\title{
PID Stabilization of Linear Neutral Time-Delay Systems in a Numerical Approach
}

\author{
Hassan Farokhi Moghadam, Nastaran Vasegh, Seyed Zeinolabedin Moussavi \\ Faculty of Electrical \& Computer Engineering, Shahid Rajaee Teacher Training University, Tehran, Iran \\ Email: hassan.farokhi89@gmail.com
}

Received August 7, 2012; revised September 7, 2012; accepted September 15, 2012

\begin{abstract}
In this paper, the stabilization of neutral time-delay systems is investigated. An efficient numerical approach is presented in an algorithm to establish results so that stability of such systems is achieved and stabilizing PID parameters are determined directly. It is based on determining the rightmost characteristic roots and Nyquist plot. The NewtonRaphson's iterative method based on Lambert W function is used for the calculation of these stabilizing roots directly from the closed-loop characteristic equation of the neutral time-delay system and then stability is checked by Nyquist plot and step response of closed-loop system. Two numerical examples are included to illustrate the effectiveness of the proposed approach.
\end{abstract}

Keywords: Neutral Time-Delay Systems; PID Controller; Stability; Iterative Method; Rightmost Characteristic Root; Nyquist Plot

\section{Introduction}

Time-delay or hereditary systems are also called systems with aftereffect or dead-time [1]. Time-delay is usually unavoidable in many mechanical and electrical systems. It often appears in control and real-world engineering systems. The presence of delays complicates the system analysis and the control design [2]. In some cases, the presence of a small delay may destabilize the system. Because of the destabilizing nature of the delayed states in a system, stability analysis of time-delay systems becomes an important area of research (see [3-7] and references therein).

As mentioned in [8], time-delay system of neutral type, where the poles lie in a band centered on the imaginary axis is the most delicate case, and the object of our present study.

In a neutral time-delay system, the time-derivative of the state depends on both the current and delayed stated and also the past derivative [9]. Stability of this type of system is playing an increasingly important role in control engineering and has received considerable attention and also has been studied extensively in the literature (see [10-14] and references therein).

PID stabilization of this type of system is the main consideration in this paper. PID (Proportional-IntegralDerivative) controller design was done by Ziegler and Nichols [15] for the first time and it is still very applicable and efficient solution to many real-world control problems with its relatively simple way of tuning. Ac- cording to a survey paper [16], more than $90 \%$ of controllers are of PID structure; even complicated control techniques also embed PID algorithms [17].

It is the most common control law for SISO systems in control engineering [18]. For this controller many design methodologies have been presented. For example, in [19], the proposed method is based on decomposing the numerator and denominator of the plant transfer function into their even and odd parts and then computing the stabilizing values of the parameters of the controller for a given time-delay system. In [20-22], a mathematical generalization of the Hermite-Biehler theorem to find all stabilizing PID controllers for systems with time-delay has been used.

In this paper, we present an efficient approach for determining the stabilizing PID controller for neutral timedelay systems. It's worthy to note that Nyquist plot and step response of closed-loop system are also used to show the correctness of our proposed stabilizing method.

The organization of this paper is as follows: In Section 2 , the main problem and the stabilizing algorithm are stated. Section 3 shows stabilization of linear neutral delayed systems. In two sub-sections we introduce rightmost characteristic root or stability determining characteristic roots and Nyquist plot which are of great role in this paper. Section 4 shows illustrating of the approach by two examples. Both MAPLE and MATLAB, the two popular mathematical softwares, are used for the programming. Simulation results will show the advantages 
of the approach. Finally, in Section 5, the main conclusions are summarized.

\section{Problem Statement}

PID is a combination of three controllers: proportional, integral and derivative controller. Thus, the PID controller can be understood as a controller that takes the present, the past, and the future of the error into consideration. The main transfer function for PID here in the $s$ domain is as follows:

$$
K(s)=k_{p}+\frac{k_{i}}{s}+k_{d} s=k_{1}+\frac{k_{2}}{s}+k_{3} s .
$$

which both equivalent forms are used in this paper. We consider the neutral time-delay systems with following general transfer function form in the $s$-domain:

$$
G(s)=\frac{1}{p(s)+q(s) \mathrm{e}^{-s \tau}}
$$

where $\tau>0$ is time-delay and $p, q$ are real polynomials. According to [8], the system is of neutral type if degrees of $p$ and $q$ are the same.

PID stabilization of this type of system is the main consideration in this paper. The stabilization is based on an algorithm which is proposed as follows.

\section{Proposed Algorithm}

At first, it's worthy to note that the algorithm is based on the known continues pole placement at [23] which is stated in following steps:

1) Initialize PID controller parameters.

2) Compute the rightmost characteristic roots for closed-loop characteristic equation of the neutral-time delay system.

3) Stop when the rightmost characteristic roots are in the left half plane and assure stability of neutral timedelay system. Then the values of PID controller parameters are chosen as stabilizing parameters. In the other case, go to the next step.

4) Compute the inverse of sensitivity matrix $\left(S_{m}\right)$ of the rightmost characteristic roots with respect to the PID controller parameters.

It's worthy to note that $S_{m}$ is similar to what defined at [20] and obtained by

$$
S_{m}=\left[s_{i, j}\right] \in R^{3 \times 3}, s_{i, j}=\frac{\partial \lambda_{i}}{\partial k_{j}}
$$

where $\lambda_{i}$ represents the rightmost characteristic roots.

5) Move the rightmost characteristic roots in the direction of the left half plane by applying a small change to the PID controller parameters as:

$$
\Delta\left(k_{1}, k_{2}, k_{3}\right)=S_{m} \delta
$$

where $\delta$ is a small change chosen optionally and it's better to be a matrix with small negative values because we want to shift the rightmost characteristic roots in the direction of the left half. It is considered here as $\delta=[-0.1-0.1-0.1]^{T}$.

6) Monitor the PID controller parameters. New PID controller parameters should be determined by adding initial values of PID controller to the values which have been obtained at step $d$. It becomes:

$$
K=\left(k_{1}, k_{2}, k_{3}\right)+\Delta\left(k_{1}, k_{2}, k_{3}\right)
$$

Now go to step $b$.

It's worthy to note that $K$ represents main stabilizing parameters of PID controller.

With respect to (1), we can rewrite (4) as

$$
\left[\begin{array}{l}
k_{p} \\
k_{i} \\
k_{d}
\end{array}\right]=\left[\begin{array}{lll}
\frac{\partial \lambda_{1}}{\partial k_{p}} & \frac{\partial \lambda_{1}}{\partial k_{i}} & \frac{\partial \lambda_{1}}{\partial k_{d}} \\
\frac{\partial \lambda_{2}}{\partial k_{p}} & \frac{\partial \lambda_{2}}{\partial k_{i}} & \frac{\partial \lambda_{2}}{\partial k_{d}} \\
\frac{\partial \lambda_{3}}{\partial k_{p}} & \frac{\partial \lambda_{3}}{\partial k_{i}} & \frac{\partial \lambda_{3}}{\partial k_{d}}
\end{array}\right]\left[\begin{array}{l}
\delta_{1} \\
\delta_{2} \\
\delta_{3}
\end{array}\right] .
$$

Advantages of this approach are shown by two illustrative examples in continuation.

\section{Stabilization of Linear Neutral Delayed Systems}

In this section, we consider the problem of designing the stabilizing PID controller for the system with the transfer function in (2). The main goal of design is to find the values of PID controller parameters such that the closedloop characteristic equation of the system is stable.

The stabilizing technique used in this paper is based on determining the rightmost characteristic roots and Nyquist plot. These two important basic principles are explained in brevity.

\subsection{The Rightmost Characteristic Roots}

Investigating the rightmost characteristic roots can be ensuring factor for stability analysis. Its importance can be seen more clearly when we know that a neutral time-delay system usually has an infinite number of roots, and it's very difficult to find out all the roots. It is usually required to find out the rightmost characteristic roots numerically [24]. In this paper the well-known Newton-Raphson's iterative method based on Lambert $\mathrm{W}$ function is used to determine the rightmost characteristic root.

In this paper we just define the following principles from $[24,25]$ which are used in determining the rightmost characteristic root.

$$
z=w \mathrm{e}^{w}, w, z \in C
$$


where $w=W(z)$ is the Lambert $W$ function. According to [24], the solution $W(z)$ has as many as infinite branches denoted by $W_{i}(z), i=0, \pm 1, \pm 2, \cdots, W_{0}(z)$ is the unique branch that is analytic at the origin $z=0$ and called the principal branch and it is used in computation of rightmost characteristic roots in this paper. It can be presented as below

$$
W_{0}(z)=\sum_{n=1}^{\infty} \frac{(-n)^{n-1}}{n !} z^{n}
$$

For fixed constants $a>0, b$ it is defined that

$$
\begin{aligned}
& F_{i}(\lambda)=a \lambda+b-W_{i}\left((a \lambda+b-\Delta(\lambda)) \mathrm{e}^{a \lambda+b}\right), \\
& i=0, \pm 1, \pm 2, \cdots
\end{aligned}
$$

where $\Delta(\lambda)$ is the characteristic equation and $F_{i}(\lambda)$ is the $i$ th branch of Lambert $W$ function. Note that, rightmost characteristic root is obtained from $F_{0}(\lambda)=0$ which is based on the main branch defined in (8). Now using Newton-Raphson's iteration method results in: [24]

$$
\lambda_{i+1}=\lambda_{i}-\frac{F\left(\lambda_{i}\right)}{F^{\prime}\left(\lambda_{i}\right)}, i=0,1,2, \cdots
$$

The iteration is stopped at step $i$ if

$$
\left|\lambda_{i}-\lambda_{i-1}\right|<\varepsilon
$$

for a given small $\varepsilon$.

Therefore $\lambda_{i}$ obtained from (10) is the rightmost characteristic root. To this end, initial guess $\left(\lambda_{0}\right)$ is chosen optionally and freely which is a complex number. In this paper initial guess and time-delay are $\lambda_{0}=0.2+3 j$ and $\tau=$ 1 , respectively.

It's worthy to note that the imaginary parts of the rightmost characteristic roots are not considered in calculations.

\subsection{Nyquist Plot}

At first we define the following theorem.

Theorem 1 [26]. A linear dynamic delay system is asymptotically stable if and only if the Nyquist diagram of

$$
W(\lambda)=\frac{\Delta(\lambda)}{(\lambda+1)^{n}}
$$

does not encircle the origin of the complex plane.

Where $\Delta(\lambda)$ is the characteristic equation and $n$ is the degree of it.

Stability is guaranteed in this paper, if Nyquist plot of

$$
W(j \omega)=\frac{\Delta\left(j \omega+\operatorname{Re} \lambda_{i}+\mu\right)}{(\lambda+1)^{n}}
$$

does not encircle the origin of the complex plane for very small $\mu>0$ [14]. In this paper $\mu$ is considered as 0.001 .
The rightmost characteristic roots are tested by putting in this theorem. If the Nyquist plot does not encounter the origin, then we will claim that rightmost characteristic roots have been computed correctly and the stability is achieved. This approach is clarified in following illustrative examples.

\section{Numerical Illustrative Examples}

To illustrate the usefulness of the proposed method, we present the following examples. Example 1 shows the application of the approach on an unstable transfer function with the second-order characteristic equation and Example 2 is a third-order time-delay system of neutral type.

\subsection{Example 1}

In this example we consider a following unstable transfer function neutral time-delay system

$$
G(s)=\frac{1}{(s+1)\left(1-\mathrm{e}^{-s}\right)}
$$

which has been considered in [8].

We will show that it can be stabilized (nominally) by a rational controller. The closed-loop characteristic equation can be rewritten as

$$
\Delta(\lambda)=\left(k_{d}+1\right) \lambda^{2}+\left(k_{p}+1\right) \lambda+k_{i}-\left(\lambda^{2}+\lambda\right) \mathrm{e}^{-\lambda}
$$

In both examples, the initial value of PID controller parameters is chosen as:

$$
\boldsymbol{K}=\left[\begin{array}{lll}
k_{p} & k_{i} & k_{d}
\end{array}\right]^{T}=\left[\begin{array}{llll}
0.2 & 0.5 & 0.1
\end{array}\right]^{T}
$$

It can be tested that the system is unstable yet and initial value should not result in stability directly.

By implicit differentiation, we have:

$$
\begin{gathered}
\frac{\partial \lambda_{i}}{\partial k_{p}}=\frac{-\lambda_{i}}{\alpha\left(\tau, \lambda_{i}\right)} \\
\frac{\partial \lambda_{i}}{\partial k_{i}}=\frac{-1}{\alpha\left(\tau, \lambda_{i}\right)} \\
\frac{\partial \lambda_{i}}{\partial k_{d}}=\frac{-\lambda_{i}^{2}}{\alpha\left(\tau, \lambda_{i}\right)}
\end{gathered}
$$

where

$$
\begin{aligned}
\alpha\left(\tau, \lambda_{i}\right)= & \tau \mathrm{e}^{-\lambda_{i} \tau} \lambda_{i}^{2}+\left(2 k_{d}+2+\tau e^{-\lambda_{i} \tau}-2 \mathrm{e}^{-\lambda_{i} \tau}\right) \lambda_{i} \\
& +k_{p}+1-\mathrm{e}^{-\lambda_{i} \tau}
\end{aligned}
$$

Now based on the step $d$ of proposed algorithm, matrix of the PID controller parameters becomes:

$$
\Delta\left(k_{p}, k_{i}, k_{d}\right)=\left[\begin{array}{lll}
-0.1121 & -0.4766 & 0.514
\end{array}\right]^{T}
$$


which cannot stabilize the system yet. So the algorithm is repeated. Based on the (5) and (6), we have:

$$
\boldsymbol{K}=\left[\begin{array}{llll}
1.9 & 0.015 & 0.614
\end{array}\right]^{T} .
$$

Now rightmost characteristic roots are obtained as (imaginary parts have been dismissed): $\lambda_{1}=-0.006718, \lambda_{2}=$ $-0.012223, \lambda_{3}=-0.012232$.

It's great that all characteristic roots are in the left half plane and can stabilize the neutral system. Therefore values in (18) become the stabilizing PID parameters. Figure 1 which is plotted based on (13) shows that Nyquist plot is in the right half plane and does not encounter the origin. According to theorem 1 the neutral system is stable. So from iteration number 2 on, the rightmost characteristic roots and consequently the stabilizing parameters, are obtained. The stabilization is also proved by the closed-loop step response which is shown in Figure 2.

Moreover, as shown in Figure 3, the curve of the real parts of the rightmost characteristic roots with respect to the delay can be produced numerically by means of the proposed algorithm, which is asymptotically stable for wide range of time delays and it is shown in Figure $\mathbf{3}$ just for $\tau \in[0,5000]$.

\subsection{Example 2}

In this example we consider another neutral time-delay system with following transfer function

$$
G(s)=\frac{1}{(s+1)\left(s+2+0.2 s \mathrm{e}^{-s}\right)}
$$

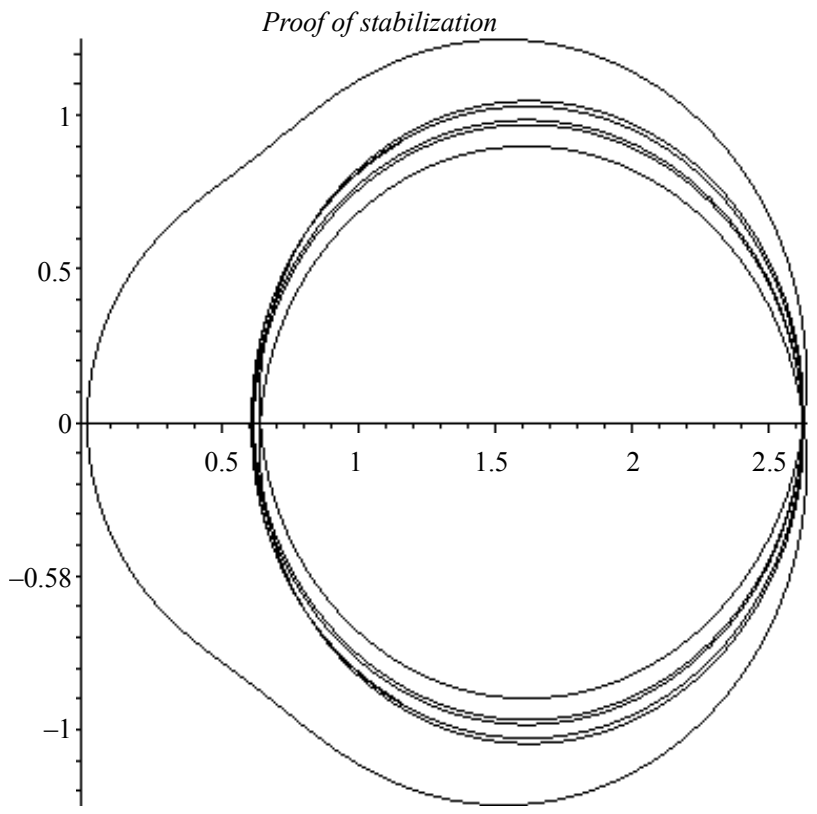

Figure 1. Proof of stabilization of Example 1 by Nyquist plot.

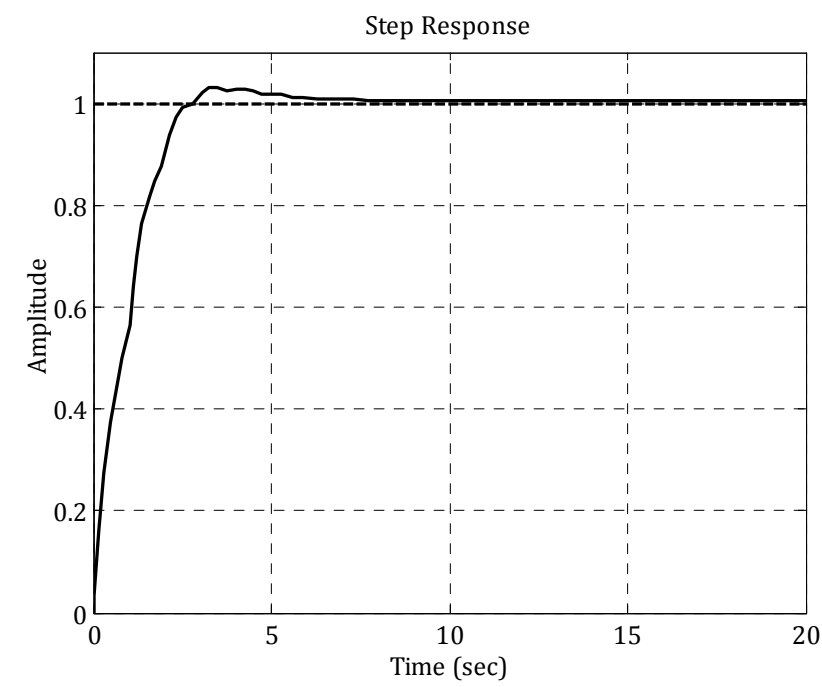

Figure 2. Step response of closed-loop system at Example 1 with PID parameters at (18).

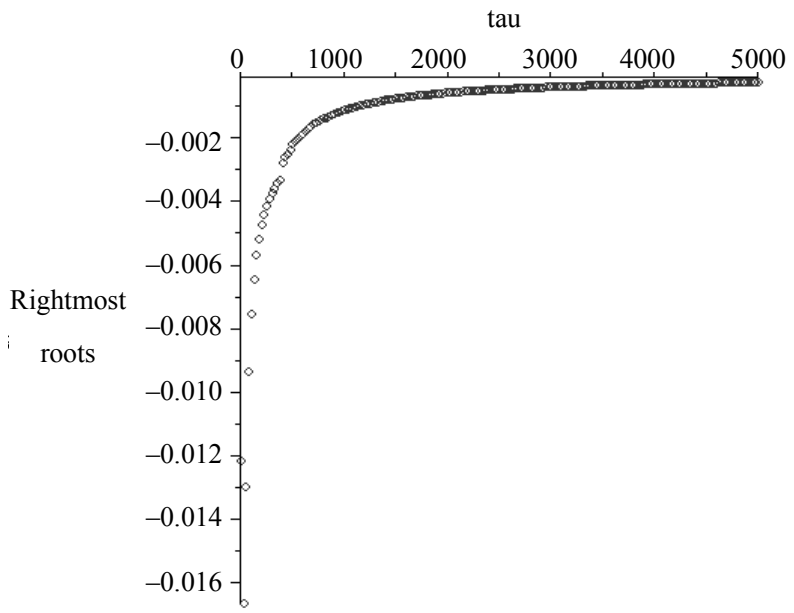

Figure 3. The stabilizing rightmost characteristic roots for wide range of time delays.

whose closed-loop characteristic equation of system can be given by

$$
\begin{aligned}
\Delta(\lambda)= & \left(1+0.2 \mathrm{e}^{-\lambda}\right) \lambda^{3}+\left(k_{d}+3+0.2 \mathrm{e}^{-\lambda}\right) \lambda^{2} \\
& +\left(k_{p}+2\right) \lambda+k_{i}
\end{aligned}
$$

It can be tested that initial values have not been resulted in stability directly. By implicit differentiation, and the procedure like example 1 , at iteration number 3 the rightmost characteristic roots and consequently the stabilizing parameters are obtained. These are obtained as $\lambda_{1}=-0.2183, \lambda_{2}=-0.3496, \lambda_{3}=-0.3505$ and

$$
\boldsymbol{K}=\left[\begin{array}{lll}
1.0904 & 0.8253 & 0.0701
\end{array}\right]^{T},
$$

respectively.

So procedure is stopped successfully. Nyquist plot in Figure 4 shows the stability of neutral system for the first 
resultant characteristic root $\left(\lambda_{1}=-0.2183\right)$. The result is similar for the others and is dismissed here for brevity.

By continuing the algorithm, at the 5th iteration, $\boldsymbol{K}=\left[\begin{array}{lll}1.61 & 1.59 & 0.01\end{array}\right]^{T}$ and at the 8th iteration, $\boldsymbol{K}=\left[\begin{array}{lll}2.2 & 2.37 & 0.0012\end{array}\right]^{T}$

have been obtained, respectively. Closed-loop step response is shown in Figure 5 for PID parameters at these different iterations.

It is worthy to note that this approach does not always stop successfully in the second or third stage. Sometime it is needed to be repeated more to get the stabilizing rightmost characteristic roots and guaranteed stability. If the desired stabilizing rightmost characteristic roots have

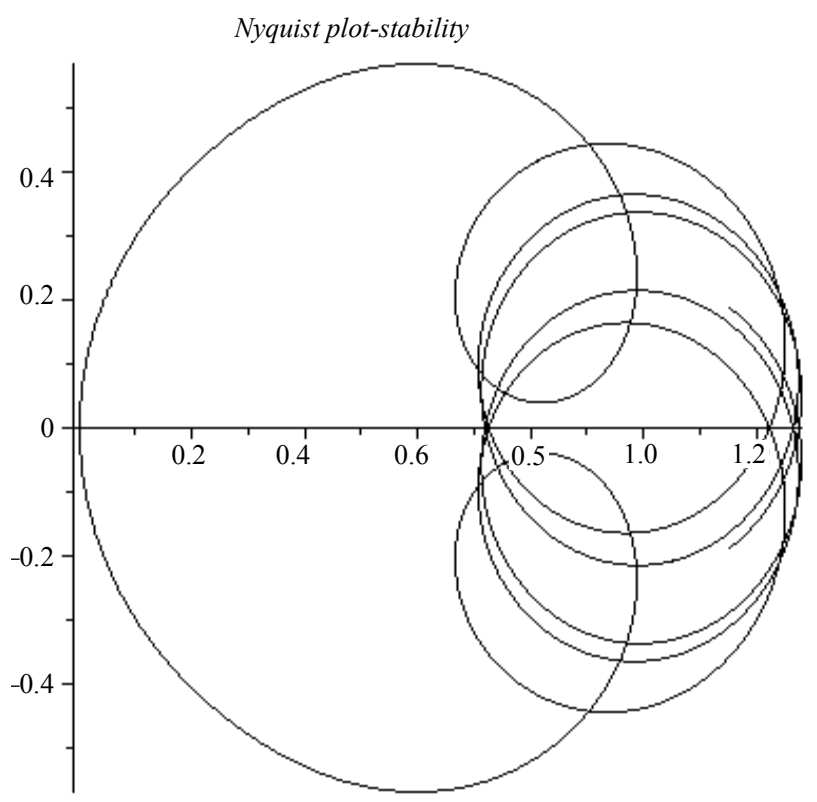

Figure 4. The Nyquist plot of Example 2 which does not encircle the origin.

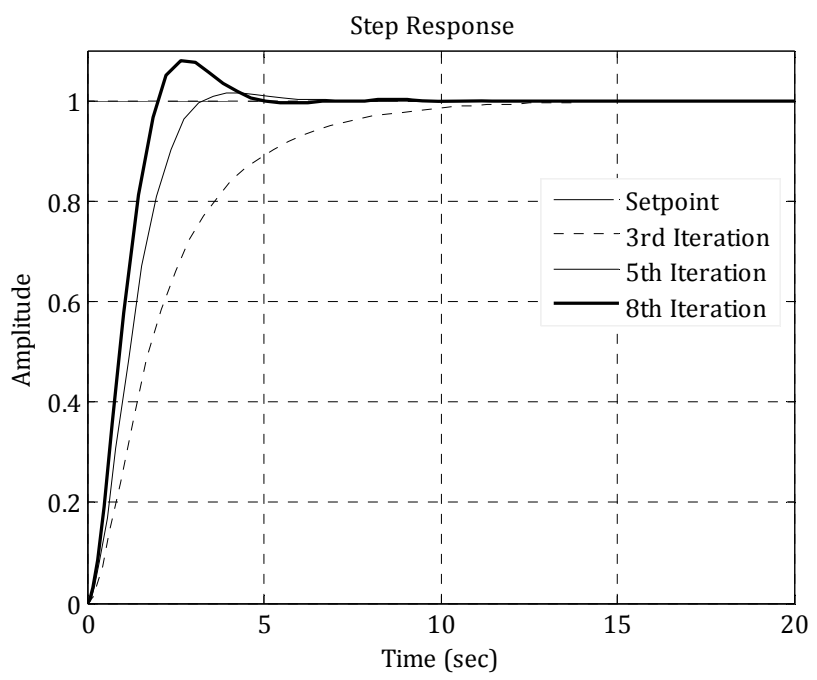

Figure 5. Step response of closed-loop system at Example 2 with PID parameters at different iterations. not been obtained after iteration number 5 , it is proposed to change the initial values of PID controller and start the algorithm from the beginning.

\section{Conclusion}

In the present paper, we presented an efficient and straightforward stabilizing PID controller design method for time-delay systems of neutral type which was free of mathematical complexities. Based on the proposed approach, stability was guaranteed if all the characteristic roots of closed-loop system had negative real parts. For determining the rightmost characteristic roots, the method was presented on the basis of Lambert $W$ function and we managed to determine the stability directly. Delay value was chosen $\tau=1$ for simplicity but the algorithm could produce a plot of the real part of the rightmost root with respect to the delay as shown in the first illustrative example. Numerical examples with time delay were presented for illustrating this approach. The results were satisfactory and stabilizing PID parameters were determined.

\section{REFERENCES}

[1] J. P. Richard, "Time-Delay Systems: An Overview of Some Recent Advances and Open Problems," Automatica, Vol. 39, No. 10, 2003, pp. 1667-1694. doi:10.1016/S0005-1098(03)00167-5

[2] J. E. Normey-Rico and E. F. Camacho, "Control of DeadTime Processes,” Springer-Verlag, London, 2007.

[3] V. B. Kolmanovskii and J. P. Richard, "Stability of Some Linear Systems with Delays," IEEE Transactions on Automatic Control, Vol. 44, No. 5, 1999, pp. 984-989. doi:10.1109/9.763213

[4] J. K. Hale and S. M. V. Lunel, "Introduction to Functional Differential Equations," Springer-Verlag, New York, 1993.

[5] L. Dugard and E. E. Verriest, "Stability and Control of Time-Delay Systems," Springer, New York, 1998. doi:10.1007/BFb0027478

[6] S. I. Niculescu and R. Lozano, "On the Passivity of Linear Delay Systems," IEEE Transactions on Automatic Control, Vol. 46, No. 3, 2001, pp. 460-464. doi:10.1109/9.911424

[7] K. Gu, V. L. Kharitonov and J. Chen, "Stability of TimeDelay Systems," Birkhauser, Boston, 2003. doi:10.1007/978-1-4612-0039-0

[8] J. R. Partingtona and C. Bonnet, " $H \infty$ and BIBO Stabilization of Delay Systems of Neutral Type," Systems \& Control Letters, Vol. 52, No. 3, 2004, pp. 283-288. doi:10.1016/j.sysconle.2003.09.014

[9] S. I. Niculescu, "Delay Effects on Stability: A Robust Control Approach,” Springer, New York, 2001.

[10] V. B. Kolmanovskii and A. D. Myshkis, "Applied Theory of Functional Differential Equations," Kluwer, Dordrecht, 
1992.

[11] V. B. Kolmanovskii and V. R. Nosov, "Stability of Functional Differential Equations," Academic Press, New York, 1986.

[12] J. H. Park and O. Kwon, "On New Stability Criterion for Delay-Differential Systems of Neutral Type," Applied Mathematics and Computation, Vol. 162, No. 2, 2005, pp. 627-637. doi:10.1016/j.amc.2004.01.001

[13] V. Chellaboina, A. Kamath and W. M. Haddad, "TimeDomain Sufficient Conditions for Stability Analysis of Linear Neutral Time-Delay Systems," Proceedings of the 2007 American Control Conference, New York, 9-13 July 2007, pp. 4917-4918.

[14] Z. H. Wang, "Numerical Stability Test of Neutral Delay Differential Equations," Hindawi Publishing Corporation, Cairo, 2008, pp. 1-10.

[15] J. G. Ziegler and N. B. Nichols, "Optimum Settings for Automatic Controllers," Transactions on ASME, Vol. 64, 1942, pp. 759-768.

[16] S. Yamamoto and I. Hashimoto, "Present Status and Future Needs: The View from Japanese Industry," Chemical Process Control-CPCIV: Proceedings of 4th International Conference on Chemical Process Control, Padre Island, 17-22 February 1991, pp. 1-28.

[17] C. Dey and R. K. Mudi, "An Improved Auto-Tuning Scheme for PID Controllers," ISA Transactions, Vol. 48, No. 4, 2009, pp. 396-408. doi:10.1016/j.isatra.2009.07.002

[18] B. Fang, "Computation of Stabilizing PID Gain Regions Based on the Inverse Nyquist Plot," Journal of Process Control, Vol. 20, No. 10, 2010, pp. 1183-1187. doi:10.1016/j.jprocont.2010.07.004
[19] N. Tan, "Computation of Stabilizing PI and PID Controllers for Processes with Time Delay," ISA Transactions, Vol. 44, No. 2, 2005, pp. 213-223. doi:10.1016/S0019-0578(07)90000-2

[20] K. W. Ho, A. Datta and S. P. Bhattacharya, "Generalizations of the Hermite-Biehler Theorem," Linear Algebra and Its Applications, Vol. 302-303, 1999, pp. 135-153. doi:10.1016/S0024-3795(99)00069-5

[21] K. W. Ho, A. Datta and S. P. Bhattacharya, "PID Stabilization of LTI Plants with Time-Delay," Proceedings of 42nd IEEE Conference on Decision and Control, Maui, 9-12 December 2003, pp. 4038-4043.

[22] G. J. Silva, A. Datta and S. P. Bhattacharyya, "PID Controllers for Time-Delay Systems," Birkhäuser, Boston, 2005.

[23] W. Michiels, K. Engelborghs, P. Vansevenant and D. Roose, "Continuous Pole Placement Method for Delay Equations," Automatica, Vol. 38, No. 5, 2002, pp. 747761. doi:10.1016/S0005-1098(01)00257-6

[24] Z. H. Wang and H. Y. Hu, "Calculation of the Rightmost Characteristic Root of Retarded Time-Delay Systems via Lambert $W$ Function," Journal of Sound and Vibration, Vol. 318, No. 4-5, 2008, pp. 757-767. doi:10.1016/j.jsv.2008.04.052

[25] R. M. Corless, G. H. Gonnet, D. E. G. Hare, D. J. Jeffrey and D. E. Knuth, "On the Lambert $W$ function," Advances in Computational Mathematics, Vol. 5, No. 4, 1996, pp. 329-359. doi:10.1007/BF02124750

[26] H. Y. Hu and Z. H. Wang, "Dynamics of Controlled Mechanical Systems with Delayed Feedback," SpringerVerlag, Berlin Heidellberg, 2002. 